\title{
Outage Probability Analysis of RIS-Assisted Wireless Networks with Von Mises Phase Errors
}

\author{
Tianxiong Wang, Mihai-Alin Badiu, Gaojie Chen, Senior Member, IEEE, and \\ Justin P. Coon, Senior Member, IEEE,
}

\begin{abstract}
In this paper, we investigate the performance of a communication link assisted by a reconfigurable intelligent surface (RIS) over line-of-sight (LOS) channels. The phase configuration of the RIS is imperfect and the phase error for each reflective element is modeled by a Von Mises distribution. First, we derive novel closed-form approximations for the lower tail distribution of the magnitude of the equivalent channel coefficient. Then, we use the obtained expressions to evaluate the outage probability and diversity order of the proposed system. Approximation errors of the proposed method are analyzed in detail. Simulation results validate the theoretical analysis is accurate at a high signal-to-noise ratio (SNR) regime.

Index Terms-Reconfigurable intelligent surface (RIS), line-
\end{abstract} of-sight (LoS), Von Mises distribution, outage probability

\section{INTRODUCTION}

Using reconfigurable intelligent surfaces (RIS) to enhance communications has been recently recognized as a promising technology for next-generation wireless networks [1]. A RIS consists of a large array of low-cost and nearly-passive reflecting elements, each element being able to adjust the phase shifts of the incident signals [2]. By a joint control over the reflecting elements, a RIS is capable of adding the reflecting signals coherently for intended users or combining signals destructively, thus improving communication performance and security [3], [4]. RIS-assisted systems were shown to offer advantages in terms of energy efficiency, deployment convenience, full-band response, low-cost, etc. [5]. Owing to its key benefits, RIS-assisted communications have the potential to support the upcoming $6 \mathrm{G}$ wireless networks [6].

Many recent papers study various performance metrics for this new technique. The authors in [7] derive an upper bound on the outage probability via central limit theorem (CLT) for a RIS-assisted network with perfect phase alignment. However, channel state information (CSI) cannot be estimated perfectly and phase shifts induced by RIS cannot be set precisely. Taking phase errors into consideration, [8][10] study bounds on the achievable data rates of RISassisted systems by using Jensen's inequality and discuss the requirements on the quantization bits to support required data rates. As a step further, the authors of [11] consider the phases errors at the RIS and conclude that the channel through the RIS is equivalent to Nakagami fading channel. In

This work was supported by EPSRC grant number EP/T02612X/1.

T. Wang, M. A. Badiu and J. P. Coon are with the Department of Engineering Science, University of Oxford, Oxford, OX1 3PJ, U.K. (e-mail: \{tianxiong.wang, mihai.badiu, justin.coon@eng.ox.ac.uk\}@eng.ox.ac.uk).

G. Chen is with 5GIC \& 6GIC, Institute for Communication Systems (ICS), University of Surrey, Guildford, GU2 7XH, United Kingdom (e-mail: gaojie.chen@surrey.ac.uk).
[12], a direct Rayleigh fading link is additionally considered, and the channel coefficient given by the reflected paths is approximated as being Gaussian distributed (according to the CLT); the distribution of the overall channel is obtained by deriving the cumulative distribution function (CDF) of the sum of a Gaussian and a Rayleigh variable. However, using the CLT is accurate only for a massive number of reflecting elements [13], [14]. Furthermore, it is shown in [13] that the CLT gives a poor approximation for the outage probability or bit error rate (BER) at high SNR regime. To circumvent these issues, the authors of [14], [15] use the moment matching method to approximate the distribution of the fading coefficient corresponding to each reflected path by a gamma distribution. Although the approaches based on the gamma distribution are accurate to some extent, they do not provide bounds or asymptotically exact results for the outage probability. In [16], the outage probability is derived in closed-form without using the approximations based on the CLT or gamma distribution. The authors use the doubleNakagami fading model and assume that phase errors are uniformly distributed within $(0,2 \pi)$. However, the assumed distribution corresponds to not having any CSI and thus the reported results can only be regarded as a lower bound on the system performance.

In this paper, we present novel results for the outage probability in a RIS-assisted communication system where the channels associated with RIS are LoS and the phases errors follow a Von Mises distribution. The set-up reflects scenarios with a dominant LoS component, e.g., a RIS equipped on an unmanned aerial vehicle (UAV). Based on experimental measurements, the channels in such scenarios usually consist of a strong LoS component and few weak scattered components. Hence, the channels can be assumed to experience no small scale fading [17]-[19]. We study the distribution of the magnitude of the equivalent-channel coefficient. In particular, we focus on the lower tail of this distribution, based on which we obtain the outage probability and diversity order for a finite number of reflecting elements. We also show that the proposed approximation is asymptotically accurate in a certain SNR region.

\section{SySTEM MOdEL}

We consider a RIS-assisted communication system consisting of a single-antenna transmitter (TX), a RIS and a singleantenna receiver (RX). It is assumed that the RIS consists of $N$ sub-wavelength-sized reflecting elements $\left(R_{1}, R_{2}, \ldots, R_{N}\right)$. Each reflecting element is of size $l \times w$ and operates in the 
far field of both TX and RX. The direct link between TX and RX is assumed to be blocked by obstacles. Denoting the normalized LoS channel coefficients of the TX-to- $R_{n}$ and the $R_{n}$-to-RX channels as $h_{1 n}=e^{j \varphi_{1 n}}$ and $h_{2 n}=e^{j \varphi_{2 n}}$, the received signal at RX can be expressed as

$$
y=\sqrt{\beta}\left(\sum_{n=1}^{N} h_{1 n} e^{j \theta_{n}} h_{2 n}\right) \sqrt{P} x+u,
$$

where $x \sim \mathcal{C N}(0,1)$ and $u \sim \mathcal{C N}\left(0, \sigma_{u}^{2}\right)$ are the transmitted symbol with normalized power and the additive white Gaussian noise (AWGN) with power $\sigma_{u}^{2}$, respectively. $P$ represents the transmit power of the TX. $\theta_{n}$ denotes the phase shift of each reflecting element. The pathloss between TX and RX through RIS in a LoS set-up was derived in [20] as

$$
\beta=\frac{G_{T} G_{R}}{(4 \pi)^{2}}\left(\frac{l w}{d_{T} d_{R}}\right)^{2} \cos ^{2}(\psi),
$$

where $G_{T}$ and $G_{R}$ are the antenna gains of TX and RX; $d_{T}$ and $d_{R}$ denote the distances between TX and RIS and RIS to RX, respectively; $\psi$ is the incident angle of the electromagnetic wave.

Ideally, to maximize the received SNR, the RIS is supposed to align the phases of all the reflecting channels by setting $\theta_{n}=\varphi_{s}-\varphi_{1 n}-\varphi_{2 n}$, where $\varphi_{s}$ is a common phase for all the channels after phase adjustment. However, in a practical system, phases errors remain due to imperfect channel knowledge ${ }^{1}$ and finite precision in phase adjustment. Mathematically, the resulting phase shift of the $n$th reflecting element is

$$
\theta_{n}=\varphi_{s}-\varphi_{1 n}-\varphi_{2 n}+\phi_{n}
$$

where $\phi_{n}, n \in\{1,2, \ldots, N\}$, is the phase error, which is modeled by mutually independent and identical Von Mises random variables. Assuming that $\phi_{n}$ follows the Von Mises distribution with zero mean and concentration parameter $\kappa$ [22], the probability density function (PDF) of $\phi_{n}$ is given by

$$
f_{\phi_{n}}(\theta)=\frac{e^{\kappa \cos (\theta)}}{2 \pi I_{0}(\kappa)}, \quad-\pi<\theta<\pi
$$

where $I_{0}(\cdot)$ is the modified Bessel function of the first kind of order zero. Substituting (3) into (1), we can rewrite the received signal as

$$
y=\sqrt{\beta P} H e^{j \varphi_{s}} x+u,
$$

where

$$
H=\sum_{n=1}^{N} e^{j \phi_{n}}
$$

Then, the received SNR can be written as

$$
\gamma=\beta|H|^{2} \gamma_{t}
$$

where $\gamma_{t}=P / \sigma_{u}^{2}$ is the transmit SNR.

In what follows, we will first treat the distribution of $|H|$ and then use the result to calculate different system performance metrics.

\footnotetext{
1 The imperfect channel state information can be assumed since the distances of the TX-to- $R_{n}$ and the $R_{n}$-to-RX links may not be measured precisely in practice [21].
}

\section{PERFORMANCE ANALYSIS}

\section{A. Preliminary Results for the Channel Distribution}

$H$ in (6) can be written in polar coordinates as

$$
H=\sum_{n=1}^{N} \cos \left(\phi_{n}\right)+j \sum_{n=1}^{N} \sin \left(\phi_{n}\right)=R \cos (\Theta)+j R \sin (\Theta),
$$

where $|H|=R$ and $\arg (H)=\Theta$. Based on [22], the PDF of $R$ can be expressed in integral form as

$$
\begin{aligned}
p_{R}(r) & =\frac{r}{I_{0}(\kappa)^{N}} I_{0}(\kappa r) \mathcal{I}(r) \\
& =\frac{r}{I_{0}(\kappa)^{N}} I_{0}(\kappa r) \int_{0}^{\infty} J_{0}(\rho r)\left(J_{0}(\rho)\right)^{N} \rho \mathrm{d} \rho,
\end{aligned}
$$

where $J_{0}(\cdot)$ denotes the Bessel function of the first kind with order zero. Then, the CDF of $R$ is given by

$$
F_{R}(r)=\int_{0}^{r} \int_{0}^{\infty} \frac{r}{I_{0}(\kappa)^{N}} I_{0}(\kappa r) J_{0}(\rho r)\left(J_{0}(\rho)\right)^{N} \rho \mathrm{d} \rho \mathrm{d} r .
$$

Changing the order of integration and evaluating the inner integral, we arrive at

$$
\begin{aligned}
F_{R}(r)= & \frac{r \kappa I_{1}(\kappa r)}{I_{0}(\kappa)^{N}} \mathcal{I}_{1}(r)+\frac{r I_{0}(\kappa r)}{I_{0}(\kappa)^{N}} \mathcal{I}_{2}(r) \\
= & \frac{r \kappa I_{1}(\kappa r)}{I_{0}(\kappa)^{N}} \int_{0}^{\infty} \frac{J_{0}(\rho)^{N} J_{0}(\rho r) \rho}{\kappa^{2}+\rho^{2}} \mathrm{~d} \rho \\
& +\frac{r I_{0}(\kappa r)}{I_{0}(\kappa)^{N}} \int_{0}^{\infty} \frac{J_{0}(\rho)^{N} J_{1}(\rho r) \rho^{2}}{\kappa^{2}+\rho^{2}} \mathrm{~d} \rho .
\end{aligned}
$$

However, (9) and (11) have infinite integration domains with oscillating integrands, which make the numerical integration difficult. In the next section, we present an approximation to the lower tail distribution of $R$.

\section{B. Analysis of the Lower Tail Distribution of $R$}

The PDF of $R$ in (9) can be analyzed asymptotically based on the Laplace's method. We denote by $\mathcal{I}(r)$ the integral in (9) and rewrite it as

$$
\mathcal{I}(r)=\int_{0}^{\infty} \rho J_{0}(\rho r) \exp \left(N \ln \left(J_{0}(\rho)\right)\right) \mathrm{d} \rho .
$$

Based on the theory of Laplace's method, the integral is dominated for large $N$ by the absolute maximum point of $\ln \left(J_{0}(\rho)\right)$. Denoting the absolute maximum point of $\ln \left(J_{0}(\rho)\right)$ by $\hat{\rho}$, we have that

$$
-\frac{J_{1}(\hat{\rho})}{J_{0}(\hat{\rho})}=0 .
$$

Therefore, the local maxima of $\ln \left(J_{0}(\rho)\right)$ are the zero points of $J_{1}(\rho)$. When $\hat{\rho}=0, \ln \left(J_{0}(\rho)\right)$ reaches its global maximum. Furthermore, $\rho J_{0}(\rho r)$ and $\ln \left(J_{0}(\rho)\right)$ can be approximated by the first terms of their Taylor series as

$$
\begin{array}{rlrl}
\rho J_{0}(\rho r) & =\rho+O\left(\rho^{3}\right), & & \rho \rightarrow 0, \\
\ln \left(J_{0}(\rho)\right) & =-\frac{\rho^{2}}{4}+O\left(\rho^{4}\right), & \rho \rightarrow 0 .
\end{array}
$$

Taking (14) into (12) and evaluating the integral yields the approximation to $p_{R}(r)$ as

$$
\tilde{p}_{R}(r)=\frac{2 r I_{0}(\kappa r)}{N I_{0}(\kappa)^{N}} .
$$


Then, the approximation to the CDF of $R$ is obtained as

$$
\tilde{F}_{R}(r)=\frac{2 r I_{1}(\kappa r)}{\kappa N I_{0}(\kappa)^{N}} \text {. }
$$

It should be noted that the CDF expression of $R$ in (11) can also be analyzed by using Laplace's method. Following the same procedure, we get the approximations to $\mathcal{I}_{1}(r)$ and $\mathcal{I}_{2}(r)$ as

$$
\tilde{\mathcal{I}}_{1}(r)=\frac{2}{\kappa^{2} N}, \quad \tilde{\mathcal{I}}_{2}(r)=\frac{4 r}{\kappa^{2} N^{2}} .
$$

Taking (17) into (11), we get the approximation to $F_{R}(r)$ as

$$
\hat{F}_{R}(r)=\frac{2 r I_{1}(\kappa r)}{\kappa N I_{0}(\kappa)^{N}}+\frac{4 r^{2} I_{0}(\kappa r)}{\kappa^{2} N^{2} I_{0}(\kappa)^{N}} .
$$

Remark 1. When $r \in\left(0, N^{\epsilon}\right)$ for $0<\epsilon<1$ and $N$ is large, we can have

$$
\begin{aligned}
\hat{F}_{R}(r) & =\tilde{F}_{R}(r)\left(1+\frac{2 r I_{0}(\kappa r)}{\kappa N I_{1}(\kappa r)}\right) \\
& =\tilde{F}_{R}(r)\left(1+O\left(\frac{1}{N^{1-\epsilon}}\right)\right), N \rightarrow \infty .
\end{aligned}
$$

Therefore, for large $N, \hat{F}_{R}(r)$ and $\tilde{F}_{R}(r)$ give the same approximation to the probability of $R$ when $R \in\left(0, N^{\epsilon}\right)$.

Remark 2. If $\kappa=0$, (15) becomes $\tilde{p}_{R}(r)=2 r / N$. Integrating over $r$, we have the asymptotic approximation to the lower tail probability of $R$ for $\kappa=0: \tilde{F}_{R}(r)=r^{2} / N$.

\section{Error Analysis}

In this section, we analyze the error of the proposed approximation and prove that $\tilde{p}_{R}(r)$ and $\tilde{F}_{R}(r)$ given in (15) and (16) are asymptotically precise in $N$ for the lower tail of the distribution of $R$. The approximation errors arise from the higher order terms of the Taylor series of $\rho J_{0}(\rho r)$ and $\ln \left(J_{0}(\rho)\right)$ :

$$
\rho J_{0}(\rho r)=\rho+A(\rho), \quad \ln \left(J_{0}(\rho)\right)=-\frac{\rho^{2}}{4}+B(\rho),
$$

where

$$
\begin{aligned}
& A(\rho)=\rho J_{0}(\rho r)-\rho=\sum_{k=1}^{\infty} a_{k} r^{2 k} \rho^{2 k+1}, \\
& B(\rho)=\ln \left(J_{0}(\rho)\right)+\frac{\rho^{2}}{4}=\sum_{j=2}^{\infty} b_{j} \rho^{2 j},
\end{aligned}
$$

where $k \geq 1$ and $j \geq 2$ are non-negative integers; $a_{k}$ and $b_{j}$ are coefficients of the series expansion. Hence, $\mathcal{I}(r)$ can be rewritten as

$$
\mathcal{I}(r)=\int_{0}^{\infty}(\rho+A(\rho)) \exp \left(-\frac{N \rho^{2}}{4}\right) \exp (N \times B(\rho)) \mathrm{d} \rho .
$$

The exponential factor $\exp (N \times B(\rho))$ can be further expanded as

$$
\exp (N \times B(\rho))=1+C(\rho)
$$

where

$$
C(\rho)=\sum_{i=1}^{\infty} \frac{1}{i !}(N \times B(\rho))^{i}=\sum_{i=1}^{\infty} \frac{1}{i !}\left(N \sum_{j=2}^{\infty} b_{j} \rho^{2 j}\right)^{i},
$$

where $i \geq 1$ is a non-negative integer. Therefore, $\mathcal{I}(r)$ can be transformed as

$$
\begin{aligned}
\mathcal{I}(r) & =\int_{0}^{\infty} \exp \left(-\frac{N \rho^{2}}{4}\right)(\rho+A(\rho))(1+C(\rho)) \mathrm{d} \rho \\
& =\mathcal{I}_{a}(r)+\mathcal{I}_{b}(r)+\mathcal{I}_{c}(r)+\mathcal{I}_{d}(r),
\end{aligned}
$$

where

$$
\begin{aligned}
& \mathcal{I}_{a}(r)=\int_{0}^{\infty} \rho \exp \left(-\frac{N \rho^{2}}{4}\right) \mathrm{d} \rho, \\
& \mathcal{I}_{b}(r)=\int_{0}^{\infty} \rho C(\rho) \exp \left(-\frac{N \rho^{2}}{4}\right) \mathrm{d} \rho, \\
& \mathcal{I}_{c}(r)=\int_{0}^{\infty} A(\rho) \exp \left(-\frac{N \rho^{2}}{4}\right) \mathrm{d} \rho, \\
& \mathcal{I}_{d}(r)=\int_{0}^{\infty} A(\rho) C(\rho) \exp \left(-\frac{N \rho^{2}}{4}\right) \mathrm{d} \rho .
\end{aligned}
$$

First, we give the following two lemmas regarding asymptotic equivalence and the convergence of the four integrals in (26).

Lemma 1. Define $f(x)$ as an integrable function of the variable $x>0$ with asymptotic expansion

$$
f(x) \sim \sum_{m=0}^{\infty} \alpha_{m} x^{m}, \quad x \rightarrow 0^{+},
$$

where $\alpha_{m}$ denotes the coefficient of the Taylor series of $f(x)$ at zero; $0^{+}$means approaching 0 from the right side of the number line. If there exists a positive number $q^{*}$, for any $q>q^{*}$, the integral $\mathcal{J}=\int_{0}^{\infty} f(x) e^{-q x^{2}} \mathrm{~d} x$ converges, then $\mathcal{J}$ has the asymptotic behavior

$$
\mathcal{J}=\int_{0}^{\infty} f(x) e^{-q x^{2}} \mathrm{~d} x \sim \sum_{m=0}^{\infty} \frac{\alpha_{m} \Gamma((1+m) / 2)}{2 q^{(1+m) / 2}}, \quad q \rightarrow \infty,
$$

where $\Gamma(\cdot)$ denotes the gamma function.

Proof: Similar to the proof of [23, Lemma 1.2].

Lemma 2. There exists a positive number $N^{*}$. For any $N>$ $N^{*}$, the four integrals $\mathcal{I}_{a}, \mathcal{I}_{b}, \mathcal{I}_{c}$ and $\mathcal{I}_{d}$ all converge.

Proof: See the Appendix A.

Next, we analyze the four equations in (26) as follows.

- We evaluate $\mathcal{I}_{a}(r)=2 / N$, which gives (15).

- Using the multinomial theorem and Lemma 1, we have

$$
\mathcal{I}_{b}(r) \sim \sum_{i \geq 1} \sum_{j \geq 2} \sum_{u \geq 0} d_{i, j, u}^{(b)} \frac{\Gamma(j i+u+1)}{N^{(j-1) i+u+1}}, \quad N \rightarrow \infty,
$$

where the non-negative integer $u$ comes from the expansion of the power of the multinomial; $d_{i, j, u}^{(b)}$ is the coefficient of each term. Therefore, we can find that $\mathcal{I}_{b}(r)=O\left(\frac{1}{N^{2}}\right), N \rightarrow \infty$.

- The asymptotic expansion of $\mathcal{I}_{c}(r)$ is given by

$$
\mathcal{I}_{c}(r) \sim \sum_{k \geq 1} d_{k}^{(c)} \frac{r^{2 k} \Gamma(k+1)}{N^{k+1}}, \quad N \rightarrow \infty .
$$

$d_{k}^{(c)}$ is the coefficient of each team. For $r \in\left(0, N^{\alpha}\right)$ and $0<\alpha<\frac{1}{2}$,

$$
\mathcal{I}_{c}(r)<\sum_{k \geq 1} \frac{d_{k}^{(c)} \Gamma(k+1)}{N^{k(1-2 \alpha)+1}}, \quad N \rightarrow \infty .
$$


Therefore, $\mathcal{I}_{c}(r)=O\left(1 / N^{2-2 \alpha}\right), N \rightarrow \infty$.

- Similarly, the asymptotic expansion of $\mathcal{I}_{d}(r)$ is

$$
\mathcal{I}_{d}(r) \sim \sum_{i \geq 1} \sum_{j \geq 2} \sum_{u \geq 0} \sum_{k \geq 1} d_{i, j, u, k}^{(d)} \frac{r^{2 k} \Gamma(j i+u+k+1)}{N^{(j-1) i+u+k+1}}, N \rightarrow \infty \text {. }
$$

$d_{i, j, u, k}^{(d)}$ is the coefficient of each term. For $r \in\left(0, N^{\alpha}\right)$ and $0<\alpha<\frac{1}{2}, \mathcal{I}_{d}(r)=O\left(1 / N^{3-2 \alpha}\right), N \rightarrow \infty$.

Therefore, we obtain that, if $r \in\left(0, N^{\alpha}\right)$ with $0<\alpha<\frac{1}{2}$,

$$
\mathcal{I}(r)=\frac{2}{N}+O\left(\frac{1}{N^{2-2 \alpha}}\right), \quad N \rightarrow \infty .
$$

This result shows that (16) gives an asymptotic approximation to the lower tail of the distribution of $R$, which is particularly important for the outage probability of the proposed system.

\section{Outage Probability and Diversity Order}

The outage probability is defined as the probability that the received SNR given by (7) falls below a threshold SNR $\bar{\gamma}$. The approximate outage probability as a function of $\gamma_{t}$ is obtained as

$$
\begin{aligned}
P_{\text {out }}\left(\gamma_{t}\right)=\tilde{F}_{R}\left(\sqrt{\frac{\bar{\gamma}}{\beta \gamma_{t}}}\right) \\
= \begin{cases}\frac{2 \sqrt{\bar{\gamma}} I_{1}\left(\kappa \sqrt{\frac{\bar{\gamma}}{\beta \gamma_{t}}}\right)}{\sqrt{\beta \gamma_{t}} \kappa N I_{0}(\kappa)^{N}}, & \text { If } \kappa>0, \gamma_{t}>\frac{\bar{\gamma}}{\beta N^{2 \alpha}} \\
\frac{\bar{\gamma}}{\beta \gamma_{t} N}, & \text { If } \kappa=0, \gamma_{t}>\frac{\bar{\gamma}}{\beta N^{2 \alpha}} .\end{cases}
\end{aligned}
$$

Remark 3. For $\kappa>0$, the outage probability is proportional to $\exp \left[-N \ln \left(I_{0}(\kappa)\right)\right] / N$. The exponential decay rate is $\ln \left(I_{0}(\kappa)\right)$, which increases with $\kappa$.

Remark 4. For $\kappa>0$, it can be proved that (34) is a decreasing function of $\kappa$ by calculating the derivative of (34) to $\kappa$. Thus, increasing the phase adjustment accuracy, i.e., $\kappa$, will lower the outage probability. Due to the limited space, detailed calculations are omitted here.

Based on [24], diversity order can be given as

$$
d=\lim _{\gamma_{t} \rightarrow \infty}-\frac{\log P_{\text {out }}}{\log \gamma_{t}}=1 .
$$

Remark 5. Diversity order of the system is one, which is due to the fact that the system model does not have $N$ independent fading channels. The proposed system model corresponds to the scenario without multipath components, e.g., RIS on UAV.

\section{NumericAl Results}

In this section, we analyze the outage probability of the system and evaluate the proposed asymptotic results by Monte Carlo (MC) simulations. In simulations, we set $d_{T}=10 \mathrm{~m}$, $d_{R}=15 \mathrm{~m}, G_{T}=G_{R}=5 \mathrm{~dB}, \psi=30^{\circ}, \bar{\gamma}=0 \mathrm{~dB}$. RIS is at the height of $8 \mathrm{~m}$. The dimension of each reflecting element is $l=22 \mathrm{~cm}, w=16 \mathrm{~cm}$, as in [25].

The outage probability versus the transmit SNR $\gamma_{t}$ for different $\kappa$ is plotted in Fig. 1 for $N=8$. As $\kappa$ increases,

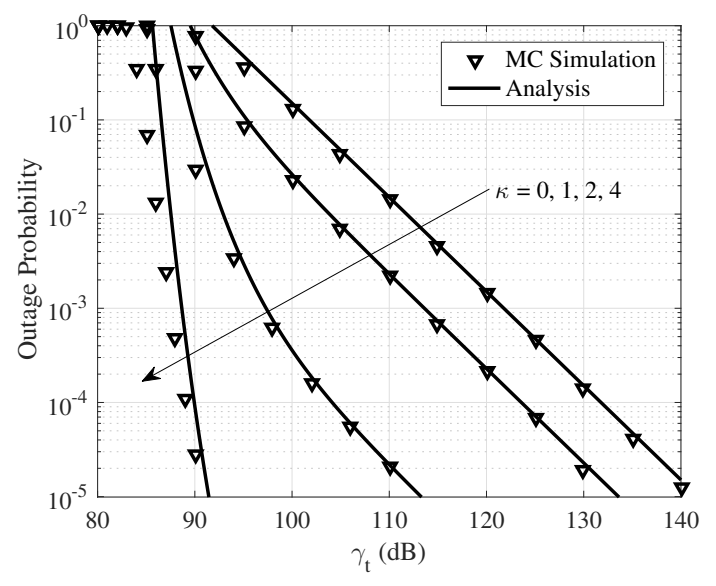

Fig. 1. Outage probability versus transmit SNR for different $\kappa$.

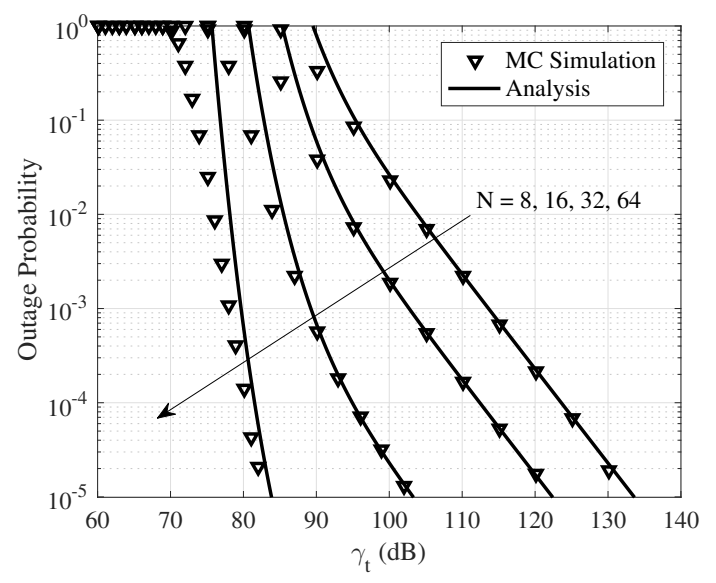

Fig. 2. Outage probability versus transmit SNR for different $N$.

the distribution becomes more concentrated about zero, which means higher phase adjustment accuracy in practical systems. As expected, increasing the phase adjustment accuracy can significantly reduce the required transmit SNR for given outage probability. For example, when the outage probability is $10^{-4}, \gamma_{t}$ is $104 \mathrm{~dB}$ and $124 \mathrm{~dB}$ for $\kappa=2$ and $\kappa=1$, respectively. The case of $\kappa=0$ is given as a benchmark, which refers to a RIS-assisted communication system that only reflects signals but does not perform any phase adjustment. We can observe that imperfect phase adjustment can bring significant performance gains compared with simple signal reflection. It also can be seen that the asymptotic expression given in (16) is very accurate in the large transmit SNR regime and diversity order of the system is one, which confirm our analysis. It is worth noting that the proposed outage probability is valid for $\gamma_{t}>\frac{\bar{\gamma}}{\beta N^{2 \alpha}}, 0<\alpha<\frac{1}{2}$. Under the current simulation setting, the valid SNR range for $N=8$ is $\gamma_{t}>95 \mathrm{~dB}$. However, when $\kappa=4$ and $\gamma_{t}>95 \mathrm{~dB}$, the outage probability is already lower than $10^{-5}$. Therefore, the analysis does not match well with the simulations for $\kappa=4$.

Fig. 2 illustrates the outage probability against $\gamma_{t}$ for different numbers of reflecting elements $N$ when $\kappa=1$. 
Again, the theoretical analysis is in good agreement with the MC simulations at high transmit SNR regime. The valid SNR range for $N=64$ is $\gamma_{t}>88 \mathrm{~dB}$, which cannot be shown in the figure. Therefore, the analysis does not approximate the simulations well for $N=64$. For larger $N$, the outage probability will decay so quickly that the valid SNR range will start at lower outage probability (smaller than $10^{-5}$ ) that cannot be shown in the figure. It can be observed that increasing the number of reflecting elements can greatly improve system performance. For example, to achieve outage probability lower than $10^{-4}$, the transmit SNR should be larger than $95 \mathrm{~dB}$ and $124 \mathrm{~dB}$ for $N=32$ and $N=8$, respectively.

\section{CONCLUSIONS}

In this paper, the outage probability and diversity order of RIS-assisted communication over LoS channels with Von Mises phase errors were analyzed. This set-up could represent imperfect phase estimation in a scenario without multi-path propagation. It was shown that increasing the number of reflecting elements and improving the accuracy of phase adjustment can significantly reduce system outage probability. Numerical results confirmed that our proposed approximate outage expression is accurate in the high transmit SNR regime. Nakagami-m channel models and the related coding gain and error probability analysis will be as the future studies.

\section{APPENDIX A}

We obtain $\mathcal{I}_{a}(r)=\frac{2}{N}$ and $\mathcal{I}_{c}(r)=\frac{2}{N}\left(\exp \left(-r^{2} / N\right)-1\right)$ [26, Eq.11.4.29]. Also since $\mathcal{I}_{a}(r)+\mathcal{I}_{b}(r)+\mathcal{I}_{c}(r)+\mathcal{I}_{d}(r)$ corresponds to the PDF in (9), which also converges, we only need to prove $\mathcal{I}_{b}(r)$ converges. $\mathcal{I}_{b}(r)$ can be rewritten as

$$
\begin{aligned}
\mathcal{I}_{b}(r) & =\int_{0}^{\infty} \rho(\exp (N \times B(\rho))-1) \exp \left(-\frac{N \rho^{2}}{4}\right) \mathrm{d} \rho \\
& =\int_{0}^{\infty} \rho J_{0}(\rho)^{N} \mathrm{~d} \rho-\frac{2}{N} .
\end{aligned}
$$

An upper bound of Bessel function of the first kind was given in [27] as $\left|J_{0}(x)\right| \leq \min \left\{1, c|x|^{-1 / 3}\right\}$, where $c=0.786$. Hence, an upper bound of the first term in (36) can be given as

$$
\int_{0}^{\infty} \rho J_{0}(\rho)^{N} \mathrm{~d} \rho<\int_{0}^{a} \rho J_{0}(\rho)^{N} \mathrm{~d} \rho+\int_{a}^{\infty} c^{N} \frac{1}{\rho^{\frac{N}{3}-1}} \mathrm{~d} \rho .
$$

We can easily learn that $\mathcal{I}_{b}(r)$ converges for $N>6$. Thus, we complete the proof of Lemma 2.

\section{REFERENCES}

[1] C. Liaskos, S. Nie et al., "A new wireless communication paradigm through software-controlled metasurfaces," IEEE Commun. Mag., vol. 56, no. 9, pp. 162-169, 2018.

[2] Q. Wu, S. Zhang et al., "Intelligent reflecting surface-aided wireless communications: A tutorial," IEEE Trans. Commun., vol. 69, no. 5, pp. 3313-3351, 2021.

[3] X. Yu, D. Xu et al., "Robust and secure wireless communications via intelligent reflecting surfaces," IEEE J. Select. Areas Commun., vol. 38, no. 11 , pp. $2637-2652,2020$.
[4] Q. Wu and R. Zhang, "Intelligent reflecting surface enhanced wireless network: Joint active and passive beamforming design," in Proc. IEEE Global Commun. Conf. (GLOBECOM), Abu Dhabi, United Arab Emirates, Dec. 2018, pp. 1-6.

[5] Z. Abdullah, G. Chen et al., "A hybrid relay and intelligent reflecting surface network and its ergodic performance analysis," IEEE Wireless Commun. Lett., vol. 9, no. 10, pp. 1653-1657, 2020.

[6] Q. Wu and R. Zhang, "Towards smart and reconfigurable environment: Intelligent reflecting surface aided wireless network," IEEE Commun. Mag., vol. 58, no. 1, pp. 106-112, 2020.

[7] D. Kudathanthirige, D. Gunasinghe, and G. Amarasuriya, "Performance analysis of intelligent reflective surfaces for wireless communication," in Proc. IEEE Int. Conf. Commun. (ICC), Dublin, Ireland, Jun. 2020, pp. 1-6.

[8] Y. Han, W. Tang et al., "Large intelligent surface-assisted wireless communication exploiting statistical CSI," IEEE Trans. Veh. Technol, vol. 68, no. 8, pp. 8238-8242, 2019.

[9] H. Zhang, B. Di et al., "Reconfigurable intelligent surfaces assisted communications with limited phase shifts: How many phase shifts are enough?" IEEE Trans. Veh. Technol, vol. 69, no. 4, pp. 4498-4502, 2020.

[10] D. Li, "Ergodic capacity of intelligent reflecting surface-assisted communication systems with phase errors," IEEE Commun. Lett., vol. 24 no. 8, pp. 1646-1650, 2020.

[11] M. Badiu and J. P. Coon, "Communication through a large reflecting surface with phase errors," IEEE Wireless Commun. Lett., vol. 9, no. 2, pp. 184-188, 2020.

[12] Q. Tao, J. Wang, and C. Zhong, "Performance analysis of intelligent reflecting surface aided communication systems," IEEE Commun. Lett., vol. 24, no. 11, pp. 2464-2468, 2020.

[13] T. Wang, G. Chen et al., "Study of intelligent reflective surface assisted communications with one-bit phase adjustments," in Proc. IEEE Global Commun. Conf. (GLOBECOM), Virtual, Dec. 2020, pp. 1-6.

[14] S. Atapattu, R. Fan et al., "Reconfigurable intelligent surface assisted two-way communications: Performance analysis and optimization," IEEE Trans. Wireless Commun., vol. 68, no. 10, pp. 6552-6567, 2020.

[15] F. A. P. de Figueiredo, M. S. P. Facina et al., "Large intelligent surfaces with discrete set of phase-shifts communicating through double-rayleigh fading channels," IEEE Access, vol. 9, pp. 20768-20787, 2021.

[16] S. A. Tegos, D. Tyrovolas et al., "On the distribution of the sum of double-nakagami-m random vectors and application in reconfigurable intelligent surfaces," 2021. [Online]. Available: https://arxiv.org/abs/2102.05591.

[17] B. Du, R. Xue et al., "Coalitional graph game for air-to-air and airto-ground cognitive spectrum sharing," IEEE Trans. Aerosp. Electron. Syst., vol. 56, no. 4, pp. 2959-2977, 2020.

[18] S. Fang, G. Chen, and Y. Li, "Joint optimization for secure intelligent reflecting surface assisted UAV networks," IEEE Wireless Communications Letters, vol. 10, no. 2, pp. 276-280, 2021.

[19] N. Gao, S. Jin et al., "Aerial RIS-assisted high altitude platform communications," IEEE Wireless Commun. Lett., pp. 1-1, 2021.

[20] Ö. Özdogan, E. Björnson, and E. G. Larsson, "Intelligent reflecting surfaces: Physics, propagation, and pathloss modeling," IEEE Wireless Commun. Lett., vol. 9, no. 5, pp. 581-585, 2019.

[21] J. A. del Peral-Rosado, R. Raulefs et al., "Survey of cellular mobile radio localization methods: From $1 \mathrm{~g}$ to 5g," IEEE Commun. Surveys Tuts., vol. 20, no. 2, pp. 1124-1148, 2017.

[22] K. V. Mardia and P. E. Jupp, Directional statistics. John Wiley \& Sons, 2009, vol. 494

[23] R. B. Paris and D. Kaminski, Asymptotics and mellin-barnes integrals. Cambridge University Press, 2001, vol. 85.

[24] Z. Wang and G. B. Giannakis, "A simple and general parameterization quantifying performance in fading channels," IEEE Trans. Commun., vol. 51, no. 8, pp. 1389-1398, 2003.

[25] W. Tang, X. Li et al., "Wireless communications with programmable metasurface: Transceiver design and experimental results," China Commun., vol. 16, no. 5, pp. 46-61, 2019.

[26] M. Abramowitz and I. A. Stegun, Handbook of mathematical functions with formulas, graphs, and mathematical tables. US Government printing office, 1964, vol. 55.

[27] L. Landau, "Bessel functions: monotonicity and bounds," Journal of the London Mathematical Society, vol. 61, no. 1, pp. 197-215, 2000. 